\title{
Basic aspects of high-power semiconductor laser simulation
}

\author{
Hans Wenzel \\ (Invited Paper)
}

\begin{abstract}
The aim of this paper is to review some of the models and solution techniques used in the simulation of high-power semiconductor lasers and to address open questions. We discuss some of the peculiarities in the description of the optical field of wide-aperture lasers. As an example, the role of the substrate as a competing waveguide in GaAs-based lasers is studied. The governing equations for the investigation of modal instabilities and filamentation effects are presented and the impact of the thermal-lensing effect on the spatiotemporal behavior of the optical field is demonstrated. We reveal the factors that limit the output power at very high injecton currents based on a numerical solution of the thermodynamic based drift-diffusion equations and elucidate the role of longitudinal spatial holeburning.
\end{abstract}

\section{INTRODUCTION}

$\mathbf{T}$ REMENDOUS advancements have been achieved in the development of high-power semiconductor lasers during the last two decades, due to improved cavity design, crystal growth, facet passivation, and device cooling technologies [1]-[6]. The output power has increased by one order of magnitude, the spectral linewidth has decreased thanks to Bragg gratings integrated into the cavity, and the beam quality has greatly improved thanks to tapered gain regions. Semiconductor lasers are under the way not longer to serve only as optical pumps for solid-state lasers but to replace them because of their ease-of-use, compactness, efficiency and high reliability. However, many of the parameters of high-power semiconductor lasers are still far from what is achievable from solid-state lasers.

For example, the reliable maximum output power is currently limited to values below $20 \mathrm{~W}$ from a $100-\mu \mathrm{m}$ stripe width device and a further increase remains an open challenge. High-power semiconductor lasers are also plagued by the appearance of multiple peaks in the lateral far- and near field profiles as well as in the optical spectrum in particular at high injection currents. These effects are caused by modal instabilities or by lasing filaments formed by a self-focusing mechanism where the refractive index locally increases in regions of high optical intensity.

In order to assess the root causes of these limitations physics-based modeling and numerical simulation is more important than ever. The aim of this paper is to review some of the models and solution techniques used in the

H. Wenzel is with the Ferdinand-Braun-Institut, Leibniz-Institut für Höchstfrequenztechnik, Gustav-Kirchhoff-Str. 4, 12489 Berlin, Germany, email: hans.wenzel@fbh-berlin.de

Manuscript received November 27, 2012; revised January 22, 2013. simulation of high-power semiconductor lasers and to address open questions.

The paper is organized as follows. We will start in Section II with a model for the optical field and introduce concepts such as the paraxial parabolic equation, roundtrip operator and modes. In Section III we discuss the modeling of the nonlinear interaction between the optical field and the injected carriers in high-power lasers leading to a non-stationary behavior of the field pattern. The simulation of the stationary electro-optical characteristics of high-power lasers using the thermodynamic based drift-diffusion (or energy transport) model will be presented in Section IV. We end with a summary and outlook.

\section{DESCRIPTION OF THE OPTICAL FIELD}

\section{A. Paraxial parabolic equation}

The optical field in edge-emitting semiconductor lasers can be well described by the paraxial parabolic equations [7]

$$
\begin{aligned}
-\frac{i k_{0} n n_{\mathrm{g}}}{c \beta_{0}} \frac{\partial E^{ \pm}}{\partial t} \mp & i \frac{\partial E^{ \pm}}{\partial z}+\frac{1}{2 \beta_{0}} \boldsymbol{\Delta}_{t} E^{ \pm} \\
& +\frac{k_{0}^{2} n^{2}-\beta_{0}^{2}}{2 \beta_{0}} E^{ \pm}+\frac{k_{0}^{2} \xi^{ \pm}}{2 \beta_{0}} E^{\mp}=0
\end{aligned}
$$

for the right and left traveling waves $E^{+}$and $E^{-}$, respectively. Eq. (1) can be derived from Maxwell equations with the Ansatz

$$
\begin{aligned}
\boldsymbol{E}\left(\boldsymbol{r}_{t}, z, t\right)=\boldsymbol{e}_{x}\left[E^{+}\left(\boldsymbol{r}_{t}, z, t\right) \mathrm{e}^{-i \beta_{0} z}\right. & \\
& \left.+E^{-}\left(\boldsymbol{r}_{t}, z, t\right) \mathrm{e}^{+i \beta_{0} z}\right] \mathrm{e}^{i \omega_{0} t}
\end{aligned}
$$

for the transverse electric field in order to remove the rapid variations with respect to the time $t$ and the coordinate $z$ along the cavity axis, and a corresponding Ansatz for the complex dielectric function,

$$
\begin{aligned}
\left.\varepsilon\left(\boldsymbol{r}_{t}, z, t, \omega\right)\right|_{\omega_{0}}= & n^{2}\left(\boldsymbol{r}_{t}, z, t\right) \\
& +\xi^{+}\left(\boldsymbol{r}_{t}\right) \mathrm{e}^{-i 2 \beta_{0} z}+\xi^{-}\left(\boldsymbol{r}_{t}\right) \mathrm{e}^{+i 2 \beta_{0} z} .
\end{aligned}
$$

In 113$), \boldsymbol{r}_{t}=(x, y)$ are the transverse coordinates, $\boldsymbol{\Delta}_{t}$ is the transverse Laplacian, $\omega_{0}$ and $\beta_{0}$ are the real-valued reference frequency and reference propagation factor, respectively, $k_{0}=$ $\omega_{0} / c=2 \pi / \lambda_{0}$ with $\lambda_{0}$ being the reference vacuum wavelength and $c$ the vacuum velocity of light, $n_{\mathrm{g}}=\partial(\omega n) /\left.\partial \omega\right|_{\omega_{0}}$ is the group index and $\xi^{ \pm}$are the Fourier coefficients of a Bragg grating integrated into the cavity. We have further assumed a TE-polarized field with $\boldsymbol{e}_{x}$ being the unity vector and that the dielectric function varies slowly with respect to 
$x$. The distribution of the complex-valued refractive index $n$ (which may include a time dependence much slower than the variation given by $\left.1 / \omega_{0}\right)$ can be written as

$$
n=n_{\mathrm{r}}+i \frac{g-\alpha}{2 k_{0}}
$$

with $g$ being the gain due to inter band transition and $\alpha$ the absorption coefficient due to intraband transitions (e.g. free carrier absorption).

The temporal dispersion of the dielectric function has been taken into account only up to the first order in the real part of the refractive index $n$, higher order dispersion and the dispersion of the imaginary part have to be properly added (see Section [III). We should note that in a numerical evaluation of (1) the factor in front of the time-derivative must be approximated by

$$
\frac{k_{0} n n_{\mathrm{g}}}{c \beta_{0}} \approx \frac{1}{v_{\mathrm{g}}}
$$

with a real-valued group velocity $v_{\mathrm{g}}$.

Eqs. (1) must be supplemented by appropriate boundary conditions. At the plane facets of the laser

$$
\begin{gathered}
E^{+}(0)-r_{0} E^{-}(0)=0, \\
E^{-}(L)-r_{L} e^{-i 2 \beta_{0} L} E^{+}(L)=0
\end{gathered}
$$

hold at $z=0$ and $z=L$, respectively. We should mention that in the paraxial approximation the facet reflectivities $r_{0}$ and $r_{L}$ are input parameters which have to be calculated in advance.

At the transverse boundary denoted by $\Gamma$ one can assume, for example, decaying fields or a perfect electric wall,

$$
\lim _{\left|\boldsymbol{r}_{t}\right| \rightarrow \infty} E^{ \pm}=0 \quad \text { or }\left.\quad E^{ \pm}\right|_{\boldsymbol{r}_{t} \in \Gamma}=0
$$

respectively. If only a part of the cross-section of the cavity is simulated, a non-reflecting or transparent boundary condition has to be used which models the fact that only outgoing waves should be present. This boundary condition can be formulated only in operator form for the general case,

$$
\left.\frac{\partial E^{ \pm}}{d \boldsymbol{n}}\right|_{\boldsymbol{r}_{t} \in \Gamma}=-\left.i \mathbf{D} E^{ \pm}\right|_{\boldsymbol{r}_{t} \in \Gamma}
$$

where $\mathbf{D}$ is the operator for a so-called Dirichlet-to-Neumann map [8]. We will consider below in Subsection [I-F]an example for a one-dimensional case. For spatially and temporally constant $n, \mathbf{D}$ can be obtained by a factorization of (1) [9]. A very popular method to implement a boundary condition of type (8) is the introduction of a so-called perfectly matched layer (PML) [10]. However, in the frequency domain it results in a large number of spurious modes [11].

\section{B. Cavity modes}

The cavity modes are time-periodic solutions of the form $E_{m}^{ \pm} \exp \left(i \Omega_{m} t\right)$ and obey the equations

$$
\begin{aligned}
\frac{k_{0} n n_{\mathrm{g}} \Omega_{m}}{c \beta_{0}} E_{m}^{ \pm} \mp i & \frac{\partial E_{m}^{ \pm}}{\partial z}+\frac{1}{2 \beta_{0}} \boldsymbol{\Delta}_{t} E_{m}^{ \pm} \\
& +\frac{k_{0}^{2} n^{2}-\beta_{0}^{2}}{2 \beta_{0}} E_{m}^{ \pm}+\frac{k_{0}^{2} \xi^{ \pm}}{2 \beta_{0}} E_{m}^{\mp}=0
\end{aligned}
$$

subject to the boundary conditions $(6)-(8)$. The non-trivial solutions of (9) may dependent on time via the time-dependence of $n$. The complex-valued relative mode frequencies $\Omega_{m}$ are the eigenvalues and the mode profiles $E_{m}^{ \pm}\left(\boldsymbol{r}_{t}, z, t\right)$ are the eigenfunctions of 9 . Some mathematical properties and their physical impact will be discussed below. The real parts of $\Omega_{m}$ give the wavelengths relative to the reference wavelength $\lambda_{0}$,

$$
\Delta \lambda_{m}=\left.\frac{d \lambda}{d \omega}\right|_{\lambda_{0}} \operatorname{Re}\left(\Omega_{m}\right)
$$

and the imaginary parts describe the damping of the modes. For a passive cavity, $\operatorname{Im}\left(\Omega_{m}\right)>0$ must hold. Lasing modes of an active cavity are distinguished by vanishing damping, $\operatorname{Im}\left(\Omega_{m}\right)=0$, due to the balance of the outcoupling and internal losses and the gain.

The cavity modes fulfill the orthogonality relation

$$
\int n n_{\mathrm{g}}\left[E_{m}^{+} E_{m^{\prime}}^{-}+E_{m}^{-} E_{m^{\prime}}^{+}\right] d x d y d z=0 \text { for } m \neq m^{\prime}
$$

which is proven in the Appendix. It is similar to corresponding orthogonality relations for cavity modes derived in [12] and [13] from Maxwell and Helmholtz equations, respectively.

Note that the integral (11) does not define a scalar product [14]. In fact, (9) defines a non-Hermitian eigenvalue problem, because the frequencies $\Omega_{m}$ are complex-valued. It is wellknown from the theory of non-Hermitian operators, that in dependence of some parameter(s) so-called "exceptional points" exist where the eigenvalues (both real and imaginary parts) cross and the eigenfunctions become identical [15]. Mode degeneracies related to exceptional points have been discovered for unstable laser cavities, $c f$. [16] and the references therein, multi-section lasers [17], [18] and complex planar waveguides [19]. Due to the mode degeneracy, at an exceptional point the system of eigenfunctions is no longer complete, but a system including the generalized eigenfunctions is, $c f .[20]$.

At the exceptional point, the modes remain orthogonal in the sense of (11), so that the integral $\int n n_{\mathrm{g}} E_{m}^{+} E_{m}^{-} d x d y d z$ vanishes. As a consequence, Petermann's $K$ factor [21]

$$
K_{m}=\left|\frac{\int n n_{\mathrm{g}}\left[\left|E_{m}^{+}\right|^{2}+\left|E_{m}^{-}\right|^{2}\right] d x d y d z}{2 \int n n_{\mathrm{g}} E_{m}^{+} E_{m}^{-} d x d y d z}\right|^{2},
$$

approaches infinity. It is known, that the $K$ factor causes an enhancement of the spontaneous emission, which results in a broadening of the spectrum of a multi-longitudinal-mode laser and the spectral linewidth of a single mode as summarized in [22]. Its time-dependence is needed to explain the dynamical behavior of multi-section lasers [23].

\section{Beam propagation method and roundtrip operator}

For a solution of 9 it is convenient to introduce the operator

$$
\mathbf{H}\left(\boldsymbol{r}_{t}, z\right)=\frac{1}{2 \beta_{0}} \boldsymbol{\Delta}_{t}+\Delta \beta
$$

with the abbreviation

$$
\Delta \beta=\frac{k_{0}^{2} n^{2}-\beta_{0}^{2}}{2 \beta_{0}} .
$$


If $\xi^{ \pm}=0$ holds (Fabry-Perot cavity), and if the index $n$ depends only on the transverse coordinates, $n=n\left(\boldsymbol{r}_{t}\right)$, the solution of $(9)$ can be formally written as

$$
E_{m}^{ \pm}\left(\boldsymbol{r}_{t}, z^{\prime}\right)=\mathrm{e}^{\mp i\left(\frac{\Omega m}{v_{g}}+\mathbf{H}\right)\left(z^{\prime}-z\right)} E_{m}^{ \pm}\left(\boldsymbol{r}_{t}, z\right) \quad \text { (FP cavity), }
$$

using the approximation (5). The numerical evaluation of (15) is the basis what is known as the beam propagation method (BPM) [24]-[26]. It has been used for the simulation of both passive [27] and active [4], [28], [29] high-power laser cavities.

For the case of a spatially and temporal constant index, $n=$ const., 15 can be evaluated exactly to yield

$$
\begin{aligned}
& E_{m}^{ \pm}\left(\boldsymbol{r}_{t}^{\prime}, z^{\prime}\right)=\mathrm{e}^{\mp i\left(\frac{\Omega_{m}}{v_{\mathrm{g}}}+\Delta \beta\right)\left(z^{\prime}-z\right)} \\
\times & \int G^{ \pm}\left(\boldsymbol{r}_{t}^{\prime}-\boldsymbol{r}_{t}, z^{\prime}-z\right) E_{m}^{ \pm}\left(\boldsymbol{r}_{t}, z\right) d x d y \quad \text { (FP cavity) }
\end{aligned}
$$

with

$$
\begin{aligned}
& G^{ \pm}\left(\boldsymbol{r}_{t}^{\prime}-\boldsymbol{r}_{t}, z^{\prime}-z\right)= \\
& \quad \Theta\left( \pm\left(z^{\prime}-z\right)\right)\left[\sqrt{ \pm \frac{i \beta_{0}}{2 \pi\left(z^{\prime}-z\right)}}\right]^{2} \mathrm{e}^{\mp \frac{i \beta_{0}\left|\boldsymbol{r}_{t}^{\prime}-\boldsymbol{r}_{t}\right|^{2}}{2\left(z^{\prime}-z\right)}}
\end{aligned}
$$

where $\Theta$ denotes the Heaviside step function. The integral equation (16) together with the propagator (17) is known as Huygen's integral in the Fresnel approximation. It has been successfully applied for the simulation of laser cavities as outlined in [30], [31], including those of semiconductor lasers [32]-[34].

Based on $(15)$ and the boundary conditions $(6)$ it is possible to construct round trip operators $\mathbf{M}^{ \pm}$. One starts at some position $z=z_{0}$ within the cavity and performs a full roundtrip. Depending whether we start into forward (+) or backward (-) directions, the eigenvalue problems

$$
\mathbf{M}^{ \pm} E_{m}^{ \pm}\left(\boldsymbol{r}_{t}, z_{0}\right)=\gamma_{m} E_{m}^{ \pm}\left(\boldsymbol{r}_{t}, z_{0}\right)
$$

are obtained with

$$
\gamma_{m}=\mathrm{e}^{i \frac{2 \Omega_{m}}{v_{\mathrm{g}}} L}
$$

being the eigenvalues. The eigenfunctions of $\mathbf{M}^{ \pm}$are the mode distributions $E_{m}^{ \pm}\left(\boldsymbol{r}_{t}, z_{0}\right)$ at the position $z_{0}$.

A very popular method for solving (18) is based on the Fox-Li approach, $c f$. [30] and the references therein. The idea is to choose a normalized, more or less arbitrary start distribution $E_{m}^{ \pm}\left(\boldsymbol{r}_{t}, z_{0}\right)$ and to apply the round trip operator $\boldsymbol{M}^{ \pm}$recurrently until one arrives (hopefully) at a steady state, i.e. a distribution that does not change its pattern in a round-trip, except for a reduction in amplitude and a phase shift which yields $\gamma_{m}$. Thereby typically the phase factor $\left.\exp \left(-2 i \operatorname{Re}(\Delta \beta) L-2 i \beta_{0} L\right)\right)$ is omitted.

The Fox-Li approach delivers the mode with the largest $\left|\gamma_{m}\right|$ if converged which is, however, not guaranteed. From a mathematical point of view, it is related to the power (also called vector or von-Mises) iteration to determine the largest eigenvalue of a matrix and the corresponding eigenvector. It is known, that the generated sequences converge only if the eigenvalue is simple and well separated from the other eigenvalues. Hence, the algorithm fails if there are cavity modes having identical or nearly identical dampings $\operatorname{Im}\left(\Omega_{m}\right)$.
Nowadays, there are more stable and efficient methods to solve (18) for passive laser cavities based on Arnoldi or Lanczos iteration. Recently, an alternative approach based on a finiteelement method for solving (9) directly, without recourse to (18), has been proposed [35].

Simulations of active laser cavities based on BPMs still utilize the Fox-Li approach [4], [36], which work quite well for single-transverse mode lasers. The approach fails for multitransverse mode lasers and at high output power if nonlinearities such as self-focusing and filamentation start to dominate, $c f$. [37] for a recent discussion of the stability of the Fox-Li approach. The association of the nonconvergence of the Fox$\mathrm{Li}$ iteration with an dynamically unstable laser behavior (as in [38]) should be done with care. Instead, for multi-mode highpower lasers, a time-dependent approach based on (1) should be preferred, although numerically more challenging.

\section{Beyond paraxial approximation}

With improving computer capabilities, direct numerical solutions of Maxwell or Helmholtz equations without recourse to the paraxial approximation become more and more feasible. For example, in [39] a scalar Helmholtz equation has been solved in the $(x, z)$ (lateral, longitudinal) plane for a high-power laser, restricted, however, to short cavities. An numerical model based on the Helmholtz operator and a firstorder time derivative obtained from Maxwell equations by separating the rapidly varying term $\exp (i \omega t)$ has been used in [40] for the simulation of a monolithically integrated masteroscillator power-amplfier. Another possibility is the expansion in terms of waveguide modes [41], [42] considered in the next Subsection.

\section{E. Waveguide modes}

We can expand the left and right traveling waves in terms of waveguide modes,

$$
E^{ \pm}\left(\boldsymbol{r}_{t}, z, t\right)=\sum_{\nu} A_{\nu}^{ \pm}(z, t) \chi_{\nu}\left(\boldsymbol{r}_{t}, z, t\right),
$$

which are solution of

$$
\boldsymbol{\Delta}_{t} \chi_{\nu}+\left[k_{0}^{2} n^{2}-\beta_{\nu}^{2}\right] \chi_{\nu}=0
$$

subject to the boundary conditions (7). The waveguide modes are exact solutions of Maxwell equations of the form $\boldsymbol{E}_{\nu}\left(\boldsymbol{r}_{t}\right) \exp \left(i \omega t-i \beta_{\nu} z\right)$ if the dielectric function is translationally invariant along $z$ [43].

Eq. 21] is in general a non-hermitian eigenvalue problem for the complex propagation factor $\beta_{\nu}$. An overview of several methods to solve [21] can be found in [25]. Expansion into waveguide modes allows an exact treatment of waveguide discontinuities such as Bragg gratings [44] or laser facets [45] in order to calculate their reflectivities. In what follows we introduce an approximate solution of 21]. 


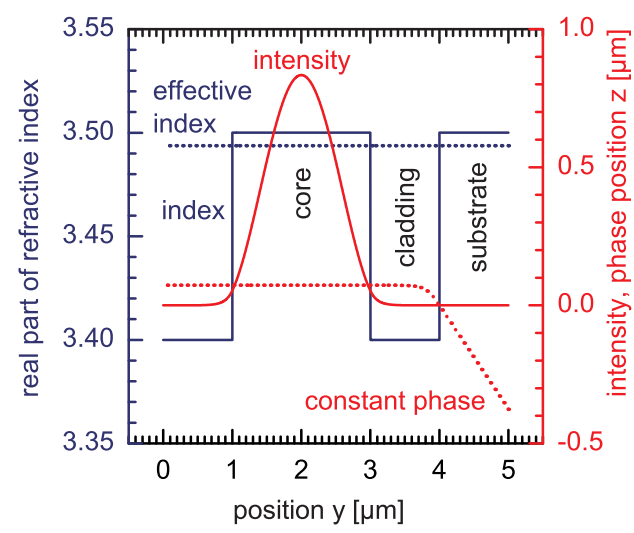

Fig. 1. Profiles of real part of refractive index(blue solid, left axis), intensity (red solid, right axis) and constant phase (red dotted, right axis) of the leaky waveguide under study. The real part of the effective index is also shown (blue dotted, left axis.)

\section{F. Effective index method}

For a nearly planar waveguide, i.e. if the mode profile $\chi(x, y)$ does not vary strongly along the $x$ direction, 21) can be solved perturbationally [46]. We choose

$$
\chi(x, y)=\Phi(x, y) \phi(x)
$$

where the dependence of $\Phi(x, y)$ on $x$ is only parametrically. If $\Phi(x, y)$ is the solution of the eigenvalue problem

$$
\frac{d^{2} \Phi}{d y^{2}}+k_{0}^{2}\left[\bar{n}^{2}-n_{\mathrm{eff}}^{2}\right] \Phi=0
$$

with

$$
\int_{-\infty}^{\infty} \Phi^{2} d y=1
$$

then $\phi(x)$ obeys the eigenvalue problem

$$
\frac{d^{2} \phi}{d x^{2}}+\left[k_{0}^{2}\left(n_{\mathrm{eff}}^{2}+\Delta n_{\mathrm{eff}}^{2}\right)-\beta^{2}\right] \phi=0
$$

with

$$
\Delta n_{\mathrm{eff}}^{2}=\int\left[n^{2}-\bar{n}^{2}\right] \Phi^{2} d y .
$$

In $23, \bar{n}(x, y)$ is a typically real-valued chosen refractiveindex distribution of a reference waveguide and $n_{\mathrm{eff}}(x)$ is the so-called effective index at position $x$. The correction $\Delta n_{\text {eff }}$ given by (26) could include e.g. modifications of the index due to carrier density and temperature effects as well as the imaginary part. The approach sketched above is called "effectiveindex method" in the semiconductor laser community. Eq. 23. is widely used in the design of high-power lasers, because it allows the optimization of the layer structure with respect to optical confinement, far-field divergence and optical losses. In what follows we will consider an example.

\section{G. Worked example}

Lasers grown on GaAs substrates, suffer from a leakage of the lasing mode into the substrate, or a coupling of the lasing mode with substrate modes because the refractive index of the substrate is typically larger than the effective index of the lasing mode. Although this effect is well-known for a long

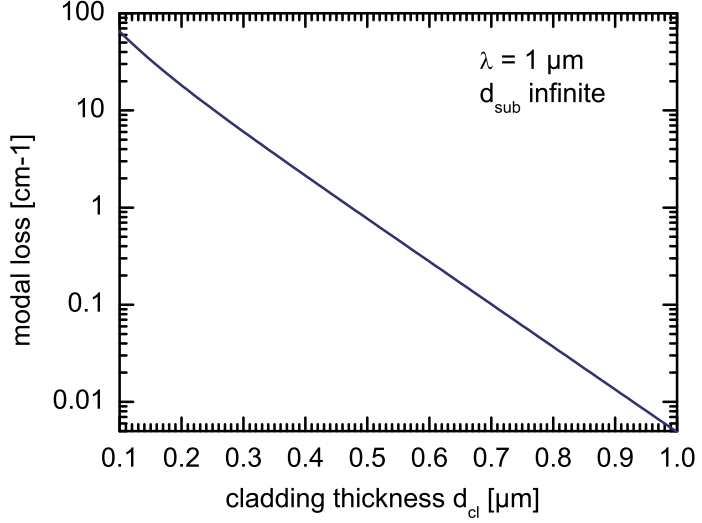

Fig. 2. Modal losses due to radiation into an infinitely thick substrate versus the thickness of the cladding layer. The vacuum wavelength is $\lambda_{0}=1 \mu \mathrm{m}$.

time [47]-[49], it has been recently re-discovered for lasers grown on GaN substrates [50]. A notably exception are lasers with a broad GaAs waveguide core and a multi-quantum well active region emitting around $1100 \mathrm{~nm}$ [51].

Assuming an infinite thick substrate, the associated losses can be determined by solving (23) subject to the condition

$$
\frac{d \Phi}{d y}=-i k_{0} \sqrt{n_{\mathrm{sub}}^{2}-n_{\mathrm{eff}}^{2}} \Phi
$$

at the boundary between cladding and substrate. Eq. 27p is an example of a boundary condition of type (8). Note that the correct branch of the complex square root function has to be chosen in order to ensure an outgoing wave. As a consequence of (27), the effective index $n_{\text {eff }}$ becomes complex valued, even though there is no absorption in the waveguide. The imaginary part of the effective index describes the loss of the modes through radiation into the substrate. If there is no absorption in the substrate or gain in an active layer, the field diverges with increasing distance from the cladding layer. If the absorption or gain is sufficiently large, this so-called leaky mode becomes a proper, albeit complex, guided mode.

Simulation tools using a PML boundary condition often fail to calculate the radiation losses of leaky waveguides correctly [52]. Using a transfer-matrix based method, the radiation loss can be exactly calculated by solving a complexvalued transcendental equation [53], [54]. We will give here the results of a worked example which can be used as a benchmark. Fig. 1 1 shows the profile of the index and the profile of the intensity of the mode with the highest effective index for a vacuum wavelength of $\lambda_{0}=1 \mu \mathrm{m}$.

Fig. 2 shows the radiation losses in dependence of the thickness of the cladding layer between waveguide core and substrate. The dependence is exponential as to be expected, except for small values of $d_{\mathrm{cl}}$, where the mode becomes strongly leaky. Due to the fact that the radiation loss increases with the effective index, a careful adjustment of the thickness of the cladding layer between substrate and waveguide core can be used to discriminate higher order vertical modes [51].

A second consequence of the optical leakage is the appear- 


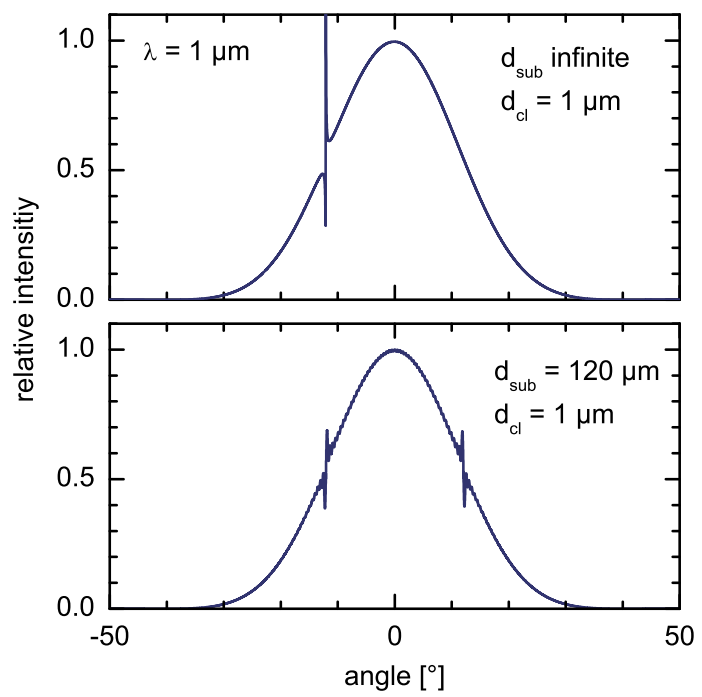

Fig. 3. Vertical profiles of the far field intensities for a substrate infinitely thick (top) and with a finite thickness (bottom).

ance of additional peak(s) in the profile of the far field intensity

$$
P_{F F}(\Theta) \propto \cos ^{2}(\Theta)\left|\int \Phi(y) \mathrm{e}^{i k_{0} \sin (\Theta) y} d y\right|^{2}
$$

in dependence on the vertical divergence angle $\Theta$. If the mode profile in the substrate

$$
\Phi(y) \propto \mathrm{e}^{-i k_{0} \sqrt{n_{\text {sub }}^{2}-n_{\text {eff }}^{2}} y}
$$

is inserted into (28), there is a resonance if

$$
\sin ^{2}\left(\Theta_{r}\right)=n_{\mathrm{sub}}^{2}-n_{\mathrm{eff}}^{2}
$$

holds. Depending whether a substrate with an infinite (Fig. 3. top) or finite (Fig. 3, bottom) thickness is assumed, one resonance or two resonances, respectively, will be observed in the far field profile. The magnitude and width of the peaks depend on the absorption in the substrate and the bottom surface roughness and metalization. The additional peak(s) in the farfield can be observed if the condition $0<n_{\text {sub }}^{2}-n_{\text {eff }}^{2}<1$ holds. If $n_{\text {sub }}^{2}-n_{\text {eff }}^{2}>1$, the internal propagation angle $\Theta_{i}=\operatorname{acos}\left(n_{\mathrm{eff}} / n_{\mathrm{sub}}\right)$, which is the angle of the normal of the line of constant phase shown in Fig. 1 with respect to the $z$ axis, is larger than the critical angle of total reflection and no peak appears.

A third consequence of the coupling of the lasing mode with substrate modes is a modulation of the intensity in the center of the waveguide core (or the optical confinement factor) in dependence on the wavelength as shown in Fig. 4. The modulation period depends strongly on the width of the waveguide core because the smaller the core the larger the wavelength dependence of the index of the mode confined to the core. This effect results in a modulation of the modal gain and hence in a corresponding modulation of the spectrum of the amplified spontaneous emission [49], [55] and the optical spectrum above threshold [56].

It should be noted that the GaAs p-contact layer causes mode coupling phenomena, too, if the thickness is not properly chosen [48].

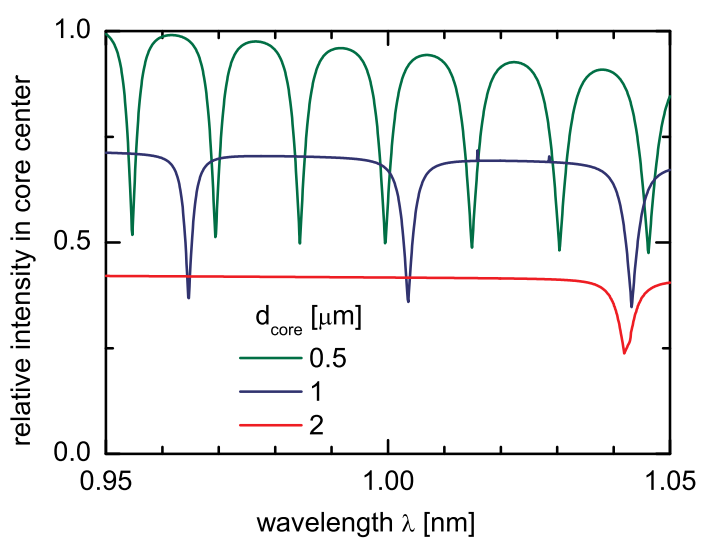

Fig. 4. Relative intensity in the waveguide core versus vacuum wavelength for different width of the waveguide core as indicated.

\section{H. Longitudinal modes}

If we insert 20 into (9) and neglect the coupling of different transverse modes due to a spatially varying group index, temporally varying index or spatially varying index perturbations, the mode amplitudes obey

$$
\frac{i \Omega_{\nu k}}{v_{\mathrm{g}, \nu}} A_{\nu k}^{ \pm} \pm \frac{d A_{\nu k}^{ \pm}}{d z}+i \Delta \beta_{\nu} A_{\nu k}^{ \pm}+i \kappa_{\nu}^{ \pm} A_{\nu k}^{\mp}=0,
$$

with the abbreviation

$$
\Delta \beta_{\nu}=\frac{1}{2 \beta_{0}}\left[\beta_{\nu}^{2}-\beta_{0}^{2}\right] .
$$

For a Fabry-Perot laser, where the coupling coefficients

$$
\kappa_{\nu}^{ \pm}=\frac{k_{0}^{2} \int \xi^{ \pm} E_{\nu}^{2} d x d y}{2 \beta_{0} \int E_{\nu}^{2} d x d y}
$$

vanish, Eq. (31) can be analytically solved subject to the boundary conditions (6). From the complex mode frequencies and 10 the wavelengths of the modes relative to the reference wavelength can be determined to

$$
\Delta \lambda_{\nu k}^{\mathrm{FP}}=-\frac{\lambda_{0}^{2}}{2 L \pi n_{\mathrm{g}, \nu}}\left[\frac{\varphi_{0}+\varphi_{L}}{2}+\pi k-L \beta_{0}-L \operatorname{Re}\left(\Delta \beta_{\nu}\right)\right]
$$

where $k$ is the longitudinal mode index and $\varphi_{0}$ and $\varphi_{L}$ are the phases of the reflectivities. The spacing between the wavelengths of different transverse modes belonging to the same longitudinal mode is approximately given by

$$
\lambda_{\nu k}-\lambda_{\nu^{\prime} k} \approx \frac{\lambda_{0}}{n_{\mathrm{g}, \nu}}\left(n_{\mathrm{mod}, \nu}-n_{\mathrm{mod}, \nu^{\prime}}\right)
$$

with $n_{\text {mod }, \nu}=\operatorname{Re}\left(\beta_{\nu}\right) / k_{0}$.

Several cases can be distinguished depending on the difference of the modal indices governing the wavelength spacing of the transversal modes. For example, in a ridge waveguide laser with a narrow ridge, the spacing of the wavelengths of the lateral modes is in the order of $1 \mathrm{~nm}$ which is much larger than that of the longitudinal modes. Hence, every lateral mode is associated with a comb of longitudinal modes which can overlap. In a broad-area laser with a wide ridge or gain region, on the other hand, the spacing of the wavelengths of the lateral modes is typically much smaller than that of the 
longitudinal modes, so that in a measured optical spectrum each longitudinal mode splits into several peaks belonging to the different lateral modes.

Assuming a 1D waveguide with width $W$ and constant real refractive index $n_{\mathrm{r}}$ surrounded by perfect electric walls, the modal indices are

$$
n_{\text {mod }, \nu}=\sqrt{n_{\mathrm{r}}^{2}-\frac{\lambda_{0}^{2}}{4 W^{2}} \nu^{2}} \quad \text { with } \quad \nu=1,2,3, \cdots .
$$

Thus the wavelength spacing with respect to the fundamental mode $\nu=1$ is given approximately

$$
\lambda_{\nu k}-\lambda_{1 k} \approx \frac{\lambda_{0}^{3}}{8 n_{\mathrm{r}} n_{\mathrm{g}} W^{2}}\left(1-\nu^{2}\right),
$$

which has been used by several authors [57], [58] for studies of broad-area lasers. It should be noted that (37) is an approximation, because it neglects the true profile of the index and the penetration of the fields into the exterior region.

\section{TIME-DEPENDENT ACTIVE CAVITY SIMULATION}

\section{A. Model equations}

In order to assess the multi-mode behavior, modal instabilities and filamentation effects of wide-aperture semiconductor lasers a time-dependent approach is the most appropriate one as outlined in Section II. Due to computer limitations, until now only the vertical-projected equations [59], [60]

$$
\begin{aligned}
- & \frac{i}{v_{\mathrm{g}, \mathrm{eff}}} \frac{\partial E^{ \pm}}{\partial t} \mp i \frac{\partial E^{ \pm}}{\partial z}+\frac{1}{2 \beta_{0}} \frac{\partial^{2} E^{ \pm}}{\partial x^{2}} \\
& +\frac{k_{0}^{2}\left[n_{\mathrm{eff}}^{2}+\Delta n_{\mathrm{eff}}^{2}\right]-\beta_{0}^{2}}{2 \beta_{0}} E^{ \pm}+\frac{k_{0}^{2} \kappa^{ \pm}}{2 \beta_{0}} E^{\mp}=F_{\text {spont }}
\end{aligned}
$$

with

$$
\kappa^{ \pm}=\frac{k_{0}^{2}}{2 \beta_{0}} \int \xi^{ \pm} \Phi^{2} d y
$$

have been dealt with. They are obtained by introducing an Ansatz like (22) into (1) and adding a Langevin source $F_{\text {spont }}$ on the rhs describing spontaneous emission. Dispersion of the imaginary part of the index (optical gain) must be additionally included to eliminate the high- $k$ instability [61], which can be done on a microscopic level [62], [63], as an effective polarization [59] modeling a Lorentzian shape of the gain, with higher order time derivatives [64], by means of a digital filter [65], or by a convolution integral [66].

Eq. (38) has to be supplemented by equations governing the carrier dynamics. In all models so far published this is done on the level of a diffusion equation for the excess carrier density $N$,

$$
\frac{\partial N}{\partial t}-\nabla[D \nabla N]+R(N)+R_{\text {stim }}=\frac{j}{e d}
$$

assuming charge neutrality in the active region. The thickness of the active region is denoted by $d$ and the rates of spontaneous (including non-radiative and radiative) and stimulated recombination by $R$ and $R_{\text {stim }}$, respectively, and $\nabla=(\partial / \partial x, \partial / \partial z)$.

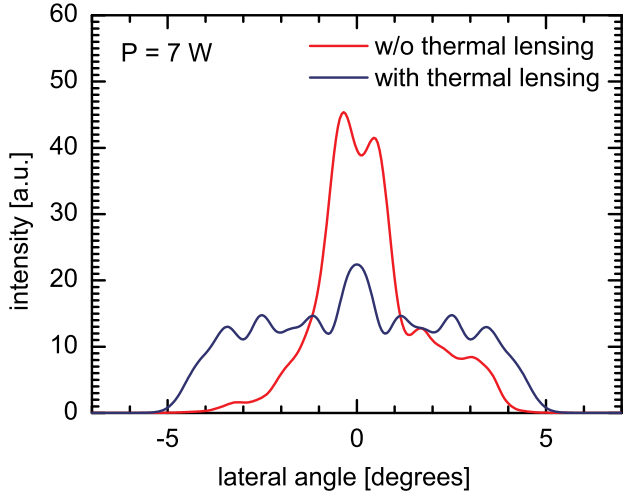

Fig. 5. Time-averaged lateral profiles of the far field intensity of a gain-guided BA DFB laser without and with the thermal lensing effect at an output power of $P=7 \mathrm{~W}$.

The current density is given by [67]

$$
j(x, z)= \begin{cases}{\left[U-U_{\mathrm{F}}(x, z)\right] / r} & \text { if } x, z \in \text { active stripe } \\ {\left[\nabla^{2} U_{\mathrm{F}}\right] / \Omega} & \text { elsewhere }\end{cases}
$$

with $U$ being the bias and $r$ and $\Omega$ being the resistivity and the sheet resistance, respectively, of the p-doped layers. The dependence of the current density within the active stripe on the Fermi voltage $U_{\mathrm{F}}$, i.e. the spacing of the electro-chemical potentials of electrons and holes, results in a preferred current injection into regions with a low carrier density, thus counteracting spatial holeburning [68], [69]. The reason is that $U_{\mathrm{F}}$ decreases and thus the difference $U-U_{\mathrm{F}}$ increases with decreasing $N$.

As shown in [70], the diffusion coefficient reads

$$
D=\mu_{p}\left(p_{0}+N\right) \frac{d U_{\mathrm{F}}}{d N}
$$

with $\mu_{p}$ being the mobility of the holes in the active layer and $p_{0}$ the equilibrium density of the holes. For Boltzmann statistics, it is given by $D=2 k_{\mathrm{B}} T \mu_{p} / e$ ( $k_{\mathrm{B}}$ Boltzmann constant, $T$ temperature, $e$ elementary charge).

The rate of stimulated recombination is given by

$$
R_{\text {stim }}=v_{\mathrm{g}, \text { eff }} g_{\text {eff }}\left[\left|E^{+}\right|^{2}+\left|E^{-}\right|^{2}\right]
$$

with the effective gain

$$
g_{\text {eff }}=\frac{1}{\operatorname{Re}\left(n_{\text {eff }}\right)} \int n_{\mathrm{r}} g \Phi^{2} d y .
$$

The output power $P_{0, L}$ at $z=0$ and $z=L$ is then given by

$$
P_{0, L}=\hbar \omega_{0} v_{\mathrm{g}, \mathrm{eff}} d\left(1-r_{0, L}^{2}\right) \int\left|E^{\mp}(x, z)\right|_{z=0, L}^{2} d x .
$$

Note, that the dispersion of the gain must be included into (43), too.

\section{B. Worked example}

The multi-peaked and not diffraction-limited lateral field profile of wide-aperture semiconductor lasers has been a long standing problem and has been investigated in the past by numerous authors [38], [71]-[74]. Although the broadening 


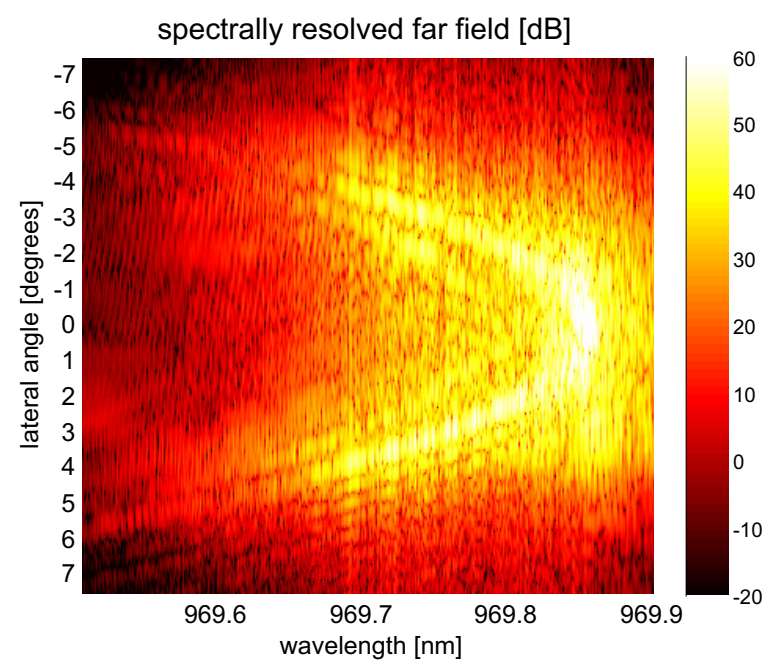

Fig. 6. Spectrally resolved far field profiles of the gain-guided BA DFB laser without the thermal lensing effect at an output power of $P=7 \mathrm{~W}$.

of the far field of continuous-wave operating lasers with increasing power can be at least partially attributed to the thermal lensing effect [58], [75], [76], a complete picture of the origin and mechanism has yet to be revealed.

One mechanism is believed to be due to carrier induced antiguiding, i.e. the reduction of the refractive index with increasing carrier density. This leads to a self-focusing mechanism because the index increases in regions of high intensity due to a depletion of the injected carrier density and can result in the formation of lasing filaments [71].

As stated in [72], the maximum local increase of the real part of the effective index is given by $\operatorname{Re}\left(\delta n_{\text {eff }}\right)=$ $\alpha_{\mathrm{H}} g_{\text {eff,th }} / 2 k_{0}$ because the carrier density can not be depleted below transparency. Hence, filamentation effects in state-ofthe-art high-power strained quantum-well lasers having a low alpha factor $\alpha_{\mathrm{H}}$ around 2 and a small threshold gain $g_{\mathrm{eff}, \mathrm{th}}$ due low internal and outcoupling losses (large cavity length) should be of less importance compared to lasers investigated earlier.

Another mechanism that could explain the multi-peaked structure and the broadening of the farfield is the simultaneous lasing of a large number of waveguide modes, originating from a built-in or thermally induced waveguide. Indeed, recent experiments reveal, that even at currents several times above threshold the lateral modes can be clearly identified by spectrally-resolved near- and farfield measurements [57], [58].

In what follows we present exemplary results obtained with the simulation tool "WIAS-LASER" [60], [77], [78] for a broad-area distributed feedback (DFB) laser [79]. The device has a cavity length of $L=3 \mathrm{~mm}$, a total width of $500 \mu \mathrm{m}$ with an active stripe width of $W=90 \mu \mathrm{m}$ and a coupling coefficient of $\kappa=3.2 \mathrm{~cm}^{-1}$.

The discretization steps are $\Delta x=0.5 \mu \mathrm{m}$ and $\Delta z=5 \mu \mathrm{m}$ into the lateral and longitudinal directions, respectively. The latter corresponds to a time step of $\Delta t=31.7$ fs. The simulation is performed over a total time window of $20 \mathrm{~ns}$, but temporal averaging and Fourier transformation is done during the last $12 \mathrm{~ns}$ thus excluding the turn-on behavior.

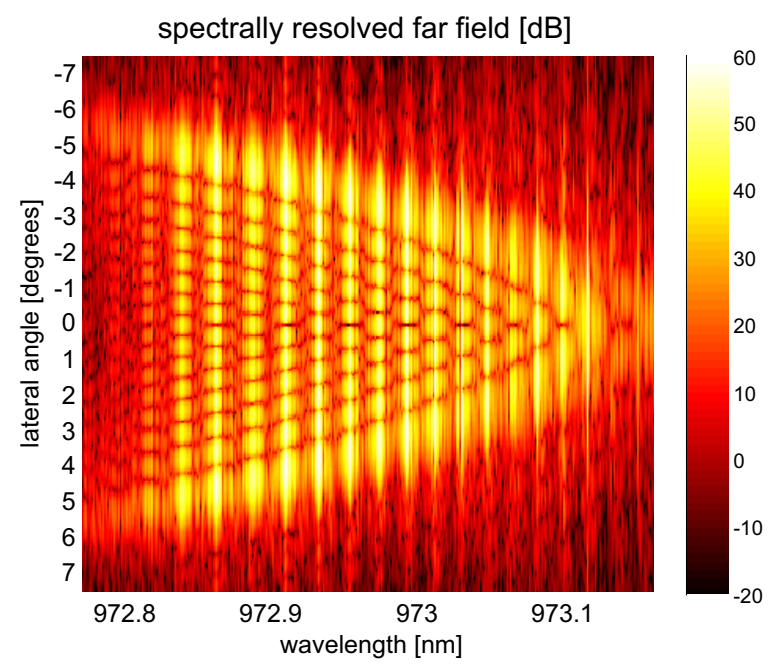

Fig. 7. Spectrally resolved far field profiles of the gain-guided BA DFB laser with the thermal lensing effect at an output power of $P=7 \mathrm{~W}$.

The dependence of the effective gain and index change on the carrier density is modeled as $g_{\mathrm{eff}}(N)=g^{\prime} \ln \left(N / N_{\mathrm{tr}}\right)$ and $\Delta n_{\text {eff }}(N)=-\sqrt{n^{\prime} N}$, respectively, with $g^{\prime}=33 \mathrm{~cm}^{-1}, N_{\mathrm{tr}}=$ $1.4 \cdot 10^{18} \mathrm{~cm}^{-3}, n^{\prime}=2.5 \cdot 10^{-25} \mathrm{~cm}^{3}$. The square root like dependence of the index on the carrier density is motivated by the result of microscopic calculations [80] and was firstly used in [28].

We will consider a device without a built-in index step emitting a temporal averaged output power of about $P=7 \mathrm{~W}$ at a current of $I=8 \mathrm{~A}$. Fig. 5 shows the temporal averaged profiles of the intensity of the far field

$$
E_{F F}(\Theta, t) \propto \int E^{-}(x, z=0, t) \mathrm{e}^{i k_{0} \sin (\Theta) x} d x
$$

for two cases, without and with a temperature induced index profile due to self-heating. The stationary temperature profile has been calculated in advance by the tool JCMsuite [76], [81]. If the thermal lens is not taken into account, the far field profile is asymmetric. This could be caused by a symmetry-breaking mechanism inherent to purely gain-guided devices [74]. With the thermal-lensing effect the far field has a symmetric shape, but is more divergent.

A deeper insight can be gained from the spectrally resolved far fields as shown in Figs. 6 and 77. In Ref. [82] the parabolic shaped dispersion curve associated with a longitudinal mode, visible in Fig. 6, was misinterpreted as a sign of lateral filamentation. We think that the opposite is true: The parabola indicates the surviving lateral mode structure, and its blurred appearance reflects the filamentation. Due to the lack of any carrier-density independent index profile, the modes react very sensitively to any changes of the carrier density, caused by local intensity fluctuations.

The index profile resulting from the self-heating stabilizes the lateral modes, so that they can be clearly identified in the spectrally resolved far field shown in Fig. 7 according to their number of nodes and antinodes. The parabolic shaped dispersion curve is caused by the fact, that according to 35 . the lasing wavelengths of the modes decrease and that the far 


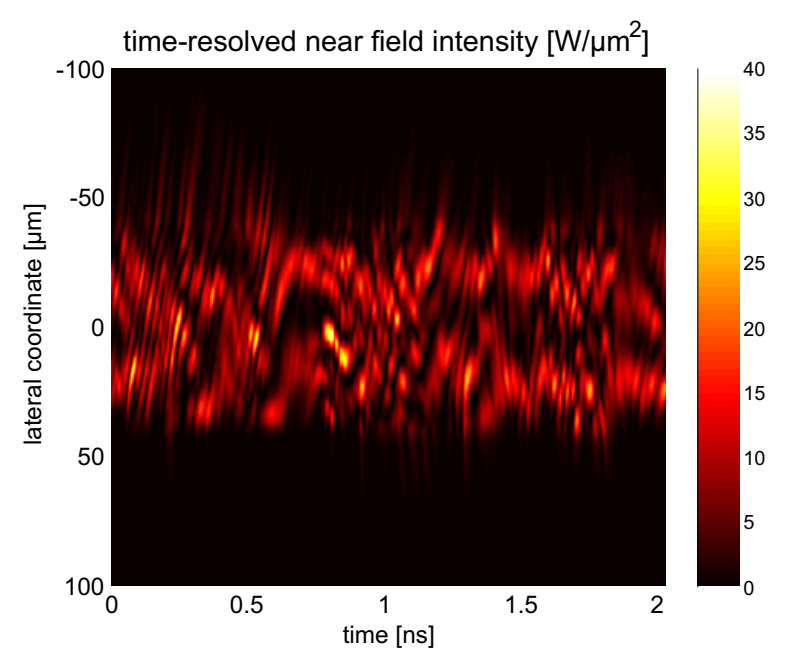

Fig. 8. Time-resolved near field profiles of the gain-guided BA DFB laser without the thermal lensing effect at an output power of $P=7 \mathrm{~W}$.

field divergence increases with rising mode index. A similar interpretation was also given in [83]. We should note, that there is only one longitudinal mode lasing due to the DFB operation.

The time-resolved near fields shown in Figs. 8 and 9 reveal the non-stationary behavior, which was already found earlier [84]. It can be interpreted to be caused by mode beating in time and space. Obviously, in the purely gain-guided case (Fig. 8) less modes are involved compared to the case with a superimposed index profile (Fig. 9). The appearance of the bright spots can be thought to be the result of a constructive interference of the lateral modes. Although the results are encouraging, further analytical and numerical investigations are needed in order to reveal the relative contributions of lateral mode and filamentary structures to the optical field of wide-aperture lasers and to obtain quantitative agreement with experimental results.

\section{STATIONARY DRIFT-DIFFUSION BASED SIMULATION}

\section{A. Basic equations}

The standard model [85]-87] for the numerical evaluation of the distributions of the electron and hole densities $N$ and $P$, temperature $T$ and electron and hole densities $\boldsymbol{j}_{n}$ and $\boldsymbol{j}_{p}$ in semiconductor lasers is the energy transport model, which consist of the Poisson equation for the electro-static potential $\varphi$,

$$
-\nabla\left[\varepsilon_{\mathrm{s}} \nabla \varphi\right]=C-P+N
$$

with $\varepsilon_{\mathrm{s}}$ being the static dielectric constant and $C$ the charged impurity density, the continuity equations

$$
\begin{aligned}
\nabla \boldsymbol{j}_{n} & =R+R_{\text {stim }} \\
-\nabla \boldsymbol{j}_{p} & =R+R_{\text {stim }}
\end{aligned}
$$

and the heat flow equation

$$
-\nabla\left[\kappa_{\mathrm{L}} \nabla T\right]=H
$$

with $\kappa_{\mathrm{L}}$ being the thermal conductivity. The various implementations of the model differ in the assumptions for the current

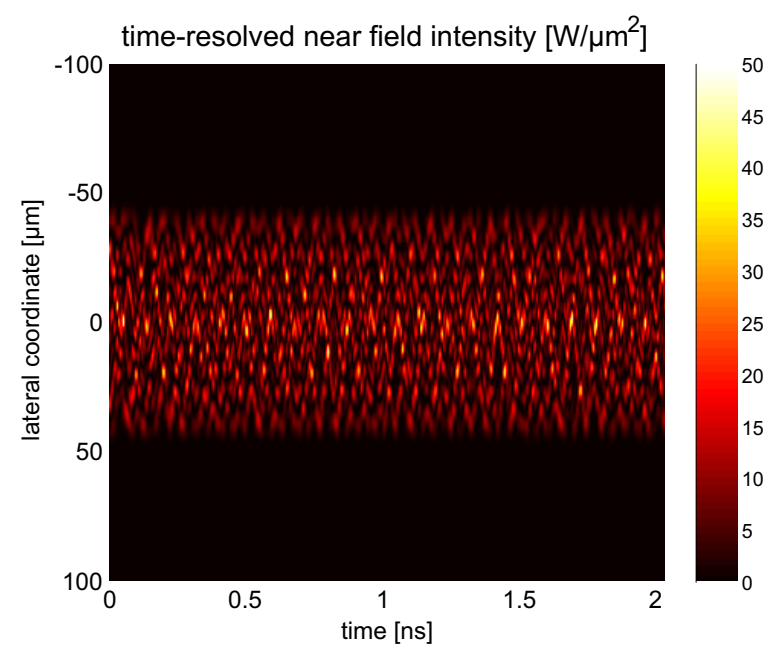

Fig. 9. Time-resolved near field profiles of the gain-guided BA DFB laser with the thermal lensing effect at an output power of $P=7 \mathrm{~W}$.

densities the $\boldsymbol{j}_{n}, \boldsymbol{j}_{p}$ and the heat source $H$. Expressions in agreement with the thermodynamic principles can be found in Ref. [88]. The rate of stimulated recombination is given here by

$$
R_{\mathrm{stim}}=\frac{n_{\mathrm{r}} g}{\hbar \omega_{0}} \sum_{\nu} \frac{P_{\nu}\left|\chi_{\nu}\right|^{2}}{n_{\mathrm{mod}, \nu} \int\left|\chi_{\nu}\right|^{2} d x d y}
$$

with $\chi_{\nu}$ obtained by solving (21).

The equations are supplemented by proper boundary conditions [88]. For example, the boundary condition for the heat flow equation reads

$$
\nu \kappa_{\mathrm{L}} \nabla T=h\left[T_{\mathrm{s}}-T\right]
$$

where $\boldsymbol{\nu}$ is the normal unit vector and $T_{\mathrm{s}}$ is the heat sink temperature. The heat transfer coefficient $h$ models the heat flow to the exterior region.

The optical output power for a given applied bias $U$ is obtained by a solution of 31. However, in Fabry-Perot lasers it is sufficient to determine the forward and backward propagating optical power $P_{\nu}^{+}=\left|A_{\nu}^{+}\right|^{2}$ and $P_{\nu}^{-}=\left|A_{\nu}^{-}\right|^{2}$, which are solutions of the equations

$$
\pm \frac{d P_{\nu}^{ \pm}}{d z}=\left[2 \operatorname{Im}\left(\beta_{\nu}+\frac{\Omega_{\nu}}{v_{\mathrm{g}, \nu}}\right)-\alpha_{0}\right] P_{\nu}^{ \pm}
$$

with $P_{\nu}=P_{\nu}^{+}+P_{\nu}^{-}$. The loss coefficient $\alpha_{0}$ includes all loss mechanisms which are not already accounted for in the imaginary part of $\beta$, such as additional internal or scattering losses. The equations (53) have to be solved subject to the boundary conditions

$$
P_{\nu}^{+}(0)=R_{0} P_{\nu}^{-}(0) \quad \text { and } \quad P_{\nu}^{-}(L)=R_{L} P_{\nu}^{+}(L) .
$$

The lasing wavelength can be approximately determined by determining the maximum of the integral $\int_{0}^{L} \operatorname{Im}\left(\beta_{\nu}+\right.$ $\left.\Omega_{\nu} / v_{\mathrm{g}, \nu}\right) d z$.

Eqs. 231 or 53 can be conveniently solved by the "Treat Power as a Parameter" (TPP) method as introduced in [68] and used in [89]. It is based on the observation, that the relative propagation factor $\Delta \beta_{\nu}$ in 31 or imaginary part $\operatorname{Im}\left(\beta_{\nu}\right)$ in 53 can be considered to be a function of the 


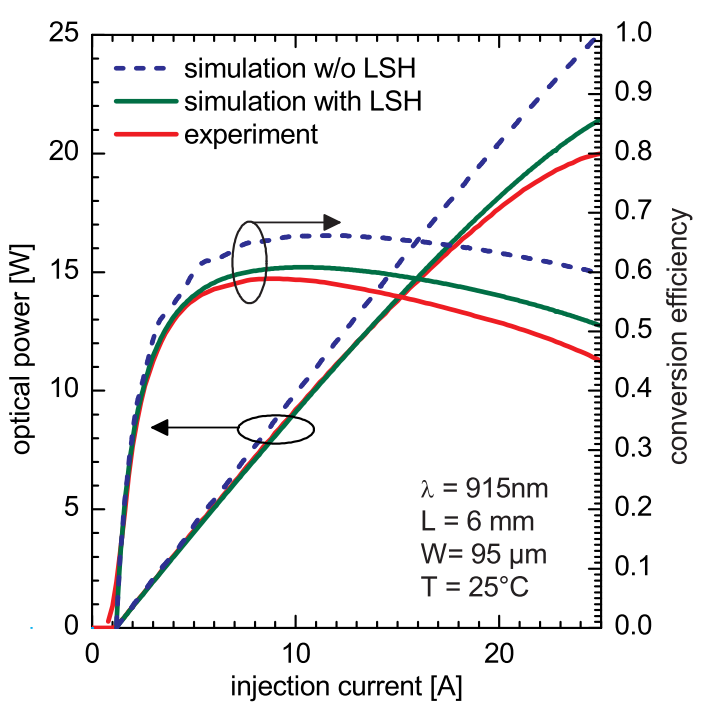

Fig. 10. Measured (solid red) and simulated optical output power (left axis) and conversion efficiency (right axis) versus injection current. The simulations were done without (dashed blue) and with (solid green) longitudinal spatial holeburning.

bias, the local power and the unknown wavelengths of the lasing and nonlasing modes. Thus, in a first step one calculates $\Delta \beta_{\nu}$ as a function of $U$, the power of the lasing waveguide mode $P_{\nu_{l}}=P_{\nu_{l}}^{+}+P_{\nu_{l}}^{-}$, and the wavelengths by solving the drift-diffusion and waveguide equations in the transverse cross section and stores the results in a in a look-up table. In a second step, $(53)$ is solved by interpolating in the look-up table. For the lasing waveguide mode, $\operatorname{Im}\left(\Omega_{\nu_{l}}\right)$ has to vanish and one has to determine the output power and the wavelength for given $U$. For the non-lasing waveguide modes one has to determine $\Omega_{\nu}$ for the given $U$ and the power distribution of the lasing mode.

Longitudinal spatial hole burning (LSH) is included automatically via the power dependence of $\operatorname{Im}\left(\beta_{\nu}\right)$ in Eq. 53). If $\operatorname{Im}\left(\beta_{\nu}\right)$ is evaluated at the average power $\bar{P}_{\nu_{l}}=\int\left[P_{\nu_{l}}^{+}+\right.$ $\left.P_{\nu_{l}}^{-}\right] d z / L$ in the cavity, the usual model neglecting LSH is recovered, because (53) is linear and can be analytically solved.

\section{B. Worked example}

In what follows we present results obtained with the simulation tool "WIAS-TeSCA" [90] for a broad-area laser. The device with a cavity length of $L=6 \mathrm{~mm}$ and an active stripe width of $W=95 \mu \mathrm{m}$ has a double quantum well (DQW) InGaAs/GaAsP active region embedded into $2.2 \mu \mathrm{m}$ wide $\mathrm{Al}_{0.30} \mathrm{Ga}_{0.70}$ As confinement and $\mathrm{Al}_{0.85} \mathrm{Ga}_{0.15} \mathrm{As}$ cladding layers. The optical confinement factor of the DQW totals $1.8 \%$. We performed a one-dimensional simulation in the transverse cross section neglecting any lateral effects as outlined in [91] and [92]. The pre-factor for the gain and the Shockley-ReadHall recombination life times ( $\tau_{n}=\tau_{p}=1.3 \mathrm{~ns}$ identical for all layers) have been fitted on the results of lengthdependent pulsed measurements of threshold current and slope efficiency of uncoated devices. The cross-sections of freecarrier absorption have been chosen to $f_{\mathrm{cn}}=3 \times 10^{-18} \mathrm{~cm}^{2}$

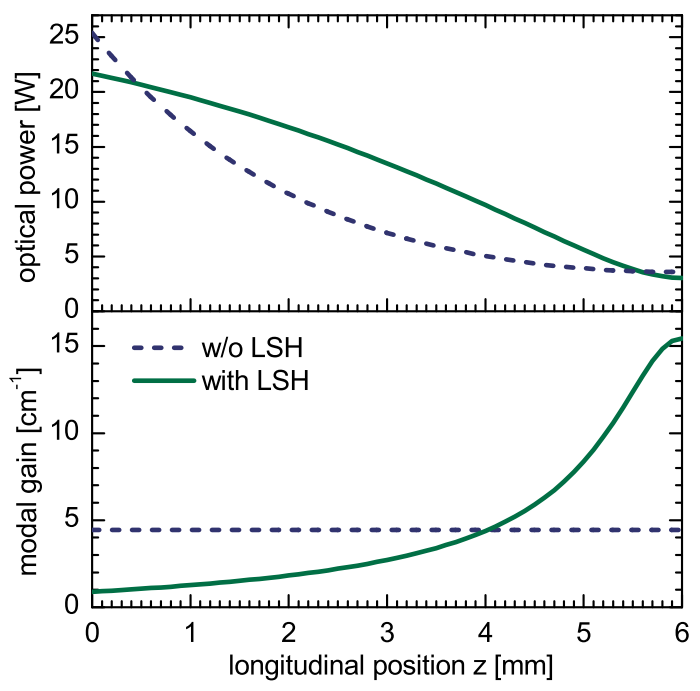

Fig. 11. Longitudinal profiles of optical power (top) and modal gain (bottom) at an injection current of $I=25$ A without (dashed blue) and with (solid green) longitudinal spatial holeburning.

and $f_{\mathrm{cp}}=7 \times 10^{-18} \mathrm{~cm}^{2}$ for electrons and holes, respectively.

Fig. 10 shows the measured and simulated power-current characteristics of the laser. Experimentally, a maximum output power of $P=20 \mathrm{~W}$ at a current of $I=25 \mathrm{~A}$ was achieved limited by thermal rollover. The experimental data were reproduced theoretically in a two-stage process with and without LSH. The reason for the rollover of the characteristics occurring already without LSH has been explained in detail in [92]. With increasing current and power, the reduction of the gain caused by the temperature rise $(\Delta T=42 \mathrm{~K}$ at $I=25$ A) must be compensated for by a corresponding increase in the carrier densities in the active region, which leads as a consequence also to an increase in the carrier densities in the confinement layers. Another effect is the bending of the quasi-Fermi energy of the holes with increasing applied bias and, as a consequence, the bending of the bandedge energies due to the voltage drop in the p-doped layers. This leads to a corresponding linear increase in the electron density with increasing distance from the active region up to $n \approx 3 \times 10^{16} \mathrm{~cm}^{-3}$. The increased carrier densities in the active region and in the bulk layers give rise to enhanced non-stimulated recombination and free-carrier absorption [93], [94].

However neglecting LSH, not only the roll-over power, but also the conversion efficiency is exaggerated which is due to the overestimation of the slope-efficiency. Good agreement between measurement and simulation is only achieved if LSH is included, as Fig. 10 reveals.

To illustrate the mechanism of LSH, Fig. 11 shows the longitudinal profiles of the optical power, the modal gain and the injected current density for the two models at a current of $I=25 \mathrm{~A}$. The rear facet with a reflectivity of $R_{L}=0.98$ is located at $\mathrm{z}=6 \mathrm{~mm}$ and the front facet with a reflectivity of $R_{0}=0.005$ at $z=0$. LSH leads to a strong depletion of the gain in the vicinity of the front facet, which is compensated by a corresponding rise of the gain at the rear facet. This results 
in a weaker increase of the power towards the front facet and hence a lower power at $z=0$, i.e. a lower output power, and is in contrast to Ref. [95] where based on an analytical model it was found that the values of the power at the facets don't dependent on the fact, whether LSH is considered or not.

It should be noted, that even with LSH there is still a small deviation in roll-over power and conversion efficiency between measurement and simulation. One reason is the neglect of the series resistances of the p-contact and the substrate in the simulation, which lead to an additional voltage drop reducing the conversion efficiency but also to additional heating reducing the roll-over power.

\section{Heterojunctions}

Heterojunctions characterized by discontinuities in the edges of the conduction and valance bands as well as abrupt changes of effective masses and mobilities require special attention. If the validity of the thermodynamic based driftdiffusion approach is assumed, the electro-chemical potentials should be continuous through the heterojunctions. The reason is, that the electro-chemical potentials and the inverse temperature can be interpreted as Lagrange multipliers in the functional for the maximization of the entropy subject to the constraints of charge and energy conservation [96].

The widely used thermionic emission theory which establishes additional conditions for the current densities [97], [98] is restricted to situations where the current essentially flows perpendicular to the junction and cannot be applied to intersections of three or more materials [99]. In highpower lasers there is no need to apply the thermionic emission theory with its difficulties because typically all heterojunctions (except at the QWs) are graded in order to avoid any drops of the electro-static potential.

\section{Quantum effects}

In the simulations presented in subsection IV-B the carriers in all layers were treated within the drift-diffusion approach, neglecting quantum effects. For more accurate simulations electrons and holes confined in active QWs must be treated in a special manner. As outlined in detail in [87], [99]-[102], the transport of the confined carriers in the in-plane directions $(x, z)$ can be described by classical drift-diffusion, but in the perpendicular direction $y$ the carriers have to be described by their quantum-mechanical wave functions. Therefore, the carriers population partitions into carriers which have sufficient energy to be be considered as unconfined and those which are confined. The scattering between both populations is described by a capture rate which has to be included into the continuity equations (48) as a recombination rate for the unconfined and a generation rate for the confined carriers.

It should be noted that the statistics of both carrier populations is governed by different electro-chemical potentials. Whereas the quasi-chemical potentials of the unconfined carriers depend directly on the bias applied to the contacts, the quasi-chemical potentials of the unconfined carriers are determined by the capture rates. Coupling of the populations takes also place via the Poisson equation (47).
Although the sketched picture where carriers are described quantum-mechanically in one direction and classically in the others has been included in several simulation tools, there are still open questions which have to be solved. The energy which partitions the carrier populations can not be always clearly defined, for example in the case of a QW located in an unbiased pn-junction. Another issue is the correct treatment of the transport in multi-quantum well structures between the QWs through the barriers.

The impact of the non-equilibrium between confined and unconfined carriers in high-power lasers on the internal efficiency, e.g., has been investigated until now using only rateequation approaches [103], not the drift-diffusion model.

\section{SUMMARY AND OUTLOOK}

We presented models for the calculation of passive cavity modes, for the investigation of the spatiotemporal behavior of the optical field and of the stationary simulation of the lightcurrent characteristics of high-power semiconductor lasers. Future work should be directed at full three-dimensional calculation of the optical field and an improvement of the physical models underlying the time-dependent active cavity simulations, including a better description of the carrier and heat transport by the energy transport model instead of the diffusion equation and stationary heat conduction equation. Finally, we would like to mention that the dependence of many material parameters and functions such as mobilities and absorption coefficients on composition, temperature and doping is not well known and needs to be improved.

\section{APPENDIX}

PROOF OF MODE ORTHOGONALITY

Let us write down (1) for 2 modes with indices $m$ and $m^{\prime}$ :

$$
\begin{aligned}
& {\left[\frac{k_{0} n n_{\mathrm{g}} \Omega_{m}}{c \beta_{0}}-i \frac{\partial}{\partial z}+\frac{1}{2 \beta_{0}} \boldsymbol{\Delta}_{\mathbf{t}}+\Delta \beta\right] E_{m}^{+}+\frac{k_{0}^{2} \xi^{+}}{2 \beta_{0}} E_{m}^{-}=0} \\
& {\left[\frac{k_{0} n n_{\mathrm{g}} \Omega_{m}}{c \beta_{0}}+i \frac{\partial}{\partial z}+\frac{1}{2 \beta_{0}} \boldsymbol{\Delta}_{\mathbf{t}}+\Delta \beta\right] E_{m}^{-}+\frac{k_{0}^{2} \xi^{-}}{2 \beta_{0}} E_{m}^{+}=0} \\
& {\left[\frac{k_{0} n n_{\mathrm{g}} \Omega_{m^{\prime}}}{c \beta_{0}}-i \frac{\partial}{\partial z}+\frac{1}{2 \beta_{0}} \boldsymbol{\Delta}_{\mathbf{t}}+\Delta \beta\right] E_{m^{\prime}}^{+}+\frac{k_{0}^{2} \xi^{+}}{2 \beta_{0}} E_{m^{\prime}}^{-}=0} \\
& {\left[\frac{k_{0} n n_{\mathrm{g}} \Omega_{m^{\prime}}}{c \beta_{0}}+i \frac{\partial}{\partial z}+\frac{1}{2 \beta_{0}} \boldsymbol{\Delta}_{\mathbf{t}}+\Delta \beta\right] E_{m^{\prime}}^{-}+\frac{k_{0}^{2} \xi^{-}}{2 \beta_{0}} E_{m^{\prime}}^{+}=0}
\end{aligned}
$$

Next 55 is multiplied with $E_{m^{\prime}}^{-}$, 56 with $E_{m^{\prime}}^{+}$, 57, with $-E_{m}^{-}$, and (58) with $-E_{m}^{+}$. Then all equations are integrated over the cavity and added to obtain

$$
\begin{gathered}
\frac{k_{0}\left(\Omega_{m}-\Omega_{m^{\prime}}\right)}{c \beta_{0}} \int n n_{\mathrm{g}}\left[E_{m}^{+} E_{m^{\prime}}^{-}+E_{m}^{-} E_{m^{\prime}}^{+}\right] d x d y d z \\
\quad-i \int \frac{\partial}{\partial z}\left[E_{m}^{+} E_{m^{\prime}}^{-}-E_{m}^{-} E_{m^{\prime}}^{+}\right] d x d y d z \\
\quad+\frac{1}{2 \beta_{0}} \int \nabla_{t}\left[E_{m^{\prime}}^{-} \nabla_{t} E_{m}^{+}+E_{m^{\prime}}^{+} \nabla_{t} E_{m}^{-}\right. \\
\left.\quad-E_{m}^{-} \nabla_{t} E_{m^{\prime}}^{+}-E_{m}^{+} \nabla_{t} E_{m^{\prime}}^{-}\right] d x d y d z=0
\end{gathered}
$$


where the derivatives have been factored out. The second and third terms vanishes due to the boundary conditions (6)-(7). Hence, the first term has to vanish, too, with leads to (11).

\section{ACKNOWLEDGMENT}

The author would like to thank R. Güther and P. Crump $(\mathrm{FBH})$ for a critical reading of the manuscript and M. Radziunas and U. Bandelow (WIAS) for providing the simulation tool "WIAS-LASER".

\section{REFERENCES}

[1] D. Welch, "A brief history of high-power semiconductor lasers," IEEE J. Sel. Topics Quantum Electron., vol. 6, no. 6, pp. 1470-1477, 2000.

[2] R. Diehl, High-power Diode Lasers: Fundamentals, Technology, Applications. Berlin Heidelberg New York: Springer, 2000, vol. 78

[3] M. Behringer, "High-power diode laser technology and characteristics," in High Power Diode Lasers, ser. Springer Series in Optical Sciences, F. Bachmann, P. Loosen, and R. Poprawe, Eds. New York: Springer, 2007, vol. 128, pp. 5-74.

[4] J. J. Lim, S. Sujecki, L. Lang, Z. Zhang, D. Paboeuf, G. Pauliat, G. Lucas-Leclin, P. Georges, R. C. I. MacKenzie, P. Bream, S. Bull, K.H. Hasler, B. Sumpf, H. Wenzel, G. Erbert, B. Thestrup, P. M. Petersen, N. Michel, M. Krakowski, and E. C. Larkins, "Design and simulation of next-generation high-power, high-brightness laser diodes," IEEE J. Sel. Topics Quantum Electron., vol. 15, no. 3, pp. 993-1008, May-Jum 2009.

[5] H. Weber, P. Loosen, and R. Poprawe, Eds., Laser Physics and Applications, Subvolume B: Laser Systems, ser. Landolt Börnstein. Berlin Heidelberg New York: Springer, 2011, vol. 3.

[6] P. Crump, O. Brox, F. Bugge, J. Fricke, C. Schultz, M. Spreemann, B. Sumpf, H. Wenzel, and G. Erbert, "High-power, high-efficiency monolithic edge-emitting GaAs based lasers with narrow spectral widths," in Advances in Semiconductor Lasers, ser. Semiconductor and Semimetals, J. J. Colemann, A. C. Bryce, and C. Jagadish, Eds. Amsterdam: Elsevier, 2012, vol. 86, ch. 2.

[7] A. E. Siegman, Lasers. Mill Valley: Univ. Science Books, 1986.

[8] X. Antoine, A. Arnold, C. Besse, M. Ehrhardt, and A. Schädle, "A review of transparent and artificial boundary conditions techniques for linear and nonlinear Schrödinger equations," Communic. Comp. Phys. vol. 4, no. 4, pp. 729-796, Oct 2008

[9] T. Moore, J. Blaschak, A. Taflove, and G. Kriegsmann, "Theory and application of radiation boundary operators," IEEE Trans. Antennas Propag., vol. 36, no. 12, pp. 1797-1812, 1988.

[10] A. Oskooi, L. Zhang, Y. Avniel, and S. Johnson, "The failure of perfectly matched layers, and towards their redemption by adiabatic absorbers," Optics Express, vol. 16, no. 15, pp. 11 376-11392, 2008.

[11] T. Tischler, Die Perfectly-Matched-Layer-Randbedingung in der FiniteDifferenzen-Methode im Frequenzbereich: Implementierung und Einsatzbereiche, ser. Innovationen mit Mikrowellen und Licht. Cuvillier, 2004

[12] H. Wenzel and H.-J. Wünsche, "An equation for the amplitudes of the modes in semiconductor lasers," IEEE J. Quantum Electron., vol. 30, no. 9, pp. 2073-2080, Sep 1994.

[13] D. V. Batrak and A. P. Bogatov, "Approximate orthogonality relation for the modes of an open cavity," Quantum Electronics, vol. 35, no. 4, pp. 356-358, Apr 2005.

[14] H. Wenzel, U. Bandelow, H.-J. Wünsche, and J. Rehberg, "Mechanisms of fast self pulsations in two-section DFB lasers," IEEE J. Quantum Electron., vol. 32, no. 1, pp. 69-78, Jan 1996.

[15] W. D. Heiss, "Exceptional points of non-hermitian operators," J. Phys. A: Math. Gen., vol. 37, no. 6, pp. 2455-2464, Feb 2004.

[16] M. V. Berry, "Mode degeneracies and the Petermann excess-noise factor for unstable lasers," J. Mod. Optics, vol. 50, no. 1, pp. 63-81, 2003.

[17] H.-J. Wünsche, U. Bandelow, H. Wenzel, and D. D. Marcenac, "Self pulsations by mode degeneracy in two-section DFB lasers," Proc. SPIE, vol. 2399, pp. 195-206, 1995 .

[18] M. Liertzer, L. Ge, A. Cerjan, A. D. Stone, H. E. Tuereci, and S. Rotter, "Pump-induced exceptional points in lasers," Phys. Rev. Lett., vol. 108, no. 17, Apr 2012.

[19] J. Ctyroky, V. Kuzmiak, and S. Eyderman, "Waveguide structures with antisymmetric gain/loss profile," Optics Express, vol. 18, no. 21, pp. 21 585-21 593, Oct 2010.
[20] J. Rehberg, H.-J. Wünsche, U. Bandelow, and H. Wenzel, "Spectral properties of a system describing fast pulsating DFB lasers," Z. Angew. Math. Mech., vol. 77, no. 1, pp. 75-77, 1997.

[21] K. Petermann, Laser Diode Modulation and Noise. Dortrecht: Kluwer Academic, 1988

[22] A. E. Siegman, "Lasers without photons - or should it be lasers with too many photons," Appl. Phys. B, vol. 60, no. 2-3, pp. 247-257, Feb-Mar 1995.

[23] U. Bandelow, R. Schatz, and H.-J. Wünsche, "A correct single-mode photon rate equation for multisection lasers," IEEE Photon. Technol. Lett., vol. 8, no. 5, pp. 614-616, May 1996.

[24] R. März, Integrated optics: Design and Modeling. Norwood, MA: Artech House, 1995.

[25] R. Scarmozzino, A. Gopinath, R. Pregla, and S. Helfert, "Numerical techniques for modeling guided-wave photonic devices," IEEE J. Sel. Topics Quantum Electron., vol. 6, no. 1, pp. 150-162, Jan-Feb 2000.

[26] T. Benson, B. B. Hu, A. Vukovic, and P. Sewell, "What is the future for beam propagation methods?" Proc. SPIE, vol. 5579, pp. 351-358, 2004.

[27] S. Sujecki, J. Wykes, P. Sewell, A. Vukovic, T. M. Benson, E. C. Larkins, L. Borruel, and I. Esquivias, "Optical properties of tapered laser cavities," IEE Proc. Optoelectron., vol. 150, no. 3, pp. 246-252, Jun 2003.

[28] L. Borruel, S. Sujecki, P. Moreno, J. Wykes, M. Krakowski, B. Sumpf, P. Sewell, S. Auzanneau, H. Wenzel, D. Rodríguez et al., "Quasi3-D simulation of high-brightness tapered lasers," IEEE J. Quantum Electron., vol. 40, no. 5, pp. 463-472, 2004.

[29] H. Odriozola, J. Tijero, L. Borruel, I. Esquivias, H. Wenzel, F. Dittmar, K. Paschke, B. Sumpf, and G. Erbert, "Beam properties of 980-nm tapered lasers with separate contacts: Experiments and simulations," IEEE J. Quantum Electron., vol. 45, no. 1, pp. 42-50, 2009.

[30] A. E. Siegman, "Laser beams and resonators: The 1960s," IEEE J. Sel. Topics Quantum Electron., vol. 6, no. 6, pp. 1380-1388, Nov-Dec 2000 .

[31] _ "Laser beams and resonators: Beyond the 1960s," IEEE J. Sel. Topics Quantum Electron., vol. 6, no. 6, pp. 1389-1399, Nov-Dec 2000.

[32] T. Fukushima, S. A. Biellak, Y. Sun, and A. E. Siegman, "Beam propagation behavior in a quasi-stadium laser diode," Optics Express, vol. 2, no. 2, pp. 21-28, Jan 1998.

[33] M. Spreemann, H. Wenzel, B. Eppich, M. Lichtner, and G. Erbert, "Novel approach to finite-aperture tapered unstable resonator lasers," IEEE J. Quantum Electron., vol. 47, no. 1, pp. 117-125, Jan 2011.

[34] M. Spreemann, J. Fricke, H. Wenzel, and G. Erbert, "Experimental and theoretical study of finite-aperture tapered unstable resonator lasers," Semicond. Sci. Technol., vol. 27, no. 10, Oct 2012

[35] K. Altmann, C. Pflaum, and D. Seider, "Third-dimensional finite element computation of laser cavity eigenmodes," Appl. Optics, vol. 43 , no. 9, pp. 1892-1901, Mar 2004.

[36] A. P. Napartovich, N. N. Elkin, V. N. Troshchieva, D. V. Vysotsky, L. J. Mawst, and D. Botez, "Comprehensive analysis of mode competition in high-power cw-operating diode lasers of the antiresonant reflecting optical waveguide (ARROW) type," IEEE J. Sel. Topics Quantum Electron., vol. 17, no. 6, pp. 1735-1744, Nov-Dec 2011.

[37] S. Sujecki, "Stability of steady-state high-power semiconductor laser models," J. Opt. Soc. Am. B, vol. 24, no. 5, pp. 1053-1060, May 2007.

[38] J. Marciante and G. Agrawal, "Nonlinear mechanisms of filamentation in broad-area semiconductor lasers," IEEE J. Quantum Electron., vol. 32, no. 4, pp. 590-596, 1996.

[39] V. P. Kalosha, K. Posilovic, and D. Bimberg, "Lateral-longitudinal modes of high-power inhomogeneous waveguide lasers," IEEE J. Quantum Electron., vol. 48, no. 2, pp. 123-128, Feb 2012.

[40] B. Berneker and C. Pflaum, "Two-dimensional dynamic simulations of DFB lasers and MOPAs," Opt. Quantum Electron., vol. 41, no. 11-13, pp. 895-902, Nov 2009

[41] R. Hocke and M. März, "Ein Berechnungsverfahren für optisch instabile Wellenleiter-Resonatoren," Frequenz, vol. 49, pp. 100-104, 1995.

[42] P. Bienstman, "Rigorous and efficient modelling of wavelength scale photonic components," Ph.D. dissertation, Univ. of Gent, 2001.

[43] J. Pomplun, S. Burger, F. Schmidt, A. Schliwa, D. Bimberg, A. Pietrzak, H. Wenzel, and G. Erbert, "Finite element simulation of the optical modes of semiconductor lasers," Phys. Stat. Sol. B, vol. 247, no. 4, pp. 846-853, Apr 2010.

[44] J. Fricke, W. John, A. Klehr, P. Ressel, L. Weixelbaum, H. Wenzel, and G. Erbert, "Properties and fabrication of high-order Bragg gratings for wavelength stabilization of diode lasers," Semicond. Sci. Technol., vol. 27 , no. 5 , p. 055009,2012 
[45] H. Derudder, D. De Zutter, and F. Olyslager, "Analysis of waveguide discontinuities using perfectly matched layers," Electron. Lett., vol. 34, no. 22, pp. 2138-2140, 1998.

[46] T. Kato, Perturbation Theory for Linear Operators. Berlin: Springer, 1995.

[47] E. V. Arzhanov, A. P. Bogatov, V. P. Konyaev, O. M. Nikitina, and V. I Shveikin, "Wave-guide properties of heterolasers based on quantumwell strained structures in InGaAs/GaAs system and characteristic properties of their gain spectra," Kvantovaya Elektronika, vol. 21, no. 7, pp. 633-639, Jul 1994.

[48] P. G. Eliseev and A. E. Drakin, "Analysis of the mode internal coupling in InGaAs/GaAs laser-diodes," Laser Physics, vol. 4, no. 3, pp. 485492, May-Jun 1994.

[49] I. A. Avrutsky, R. Gordon, R. Clayton, and J. M. Xu, "Investigations of the spectral characteristics of 980-nm InGaAs-GaAs-AlGaAs lasers," IEEE J. Quantum Electron., vol. 33, no. 10, pp. 1801-1809, Oct 1997.

[50] V. Laino, F. Roemer, B. Witzigmann, C. Lauterbach, U. T. Schwarz, C. Rumbolz, M. O. Schillgalies, M. Furitsch, A. Lell, and V. Haerle, "Substrate modes of (Al,In)GaN semiconductor laser diodes on $\mathrm{SiC}$ and GaN substrates," IEEE J. Quantum Electron., vol. 43, no. 1, pp. 16-24, Jan-Feb 2007.

[51] G. Erbert, F. Bugge, J. Fricke, P. Ressel, R. Staske, B. Sumpf, H. Wenzel, M. Weyers, and G. Tränkle, "High power high-efficiency 1150-nm quantum-well laser," IEEE J. Sel. Topics Quantum Electron., vol. 11, no. 5, pp. 1217-1222, Sep-Oct 2005.

[52] P. Bienstman, S. Selleri, L. Rosa, H. P. Uranus, W. C. L. Hopman, R. Costa, A. Melloni, L. C. Andreani, J. P. Hugonin, P. Lalanne, D. Pinto, S. S. A. Obayya, M. Dems, and K. Panajotov, "Modelling leaky photonic wires: A mode solver comparison," Opt. Quantum Electron., vol. 38, no. 9-11, pp. 731-759, Jul 2006.

[53] H. Wenzel and H. Wünsche, "A model for the calculation of the threshold current of SCH-MQW-SAS lasers," phys. stat. sol. (a), vol. 120, no. 2, pp. 661-673, 1990.

[54] J. Petracek and K. Singh, "Determination of leaky modes in planar multilayer waveguides," IEEE Photon. Technol. Lett., vol. 14, no. 6 , pp. 810-812, 2002.

[55] B. Witzigmann, V. Laino, F. Roemer, C. Lauterbach, U. T. Schwarz, C. Rumbolz, M. O. Schillgalies, A. Lell, U. Strauss, and V. Hrle, "Analysis of substrate modes in GaN/InGaN lasers," Proc. SPIE, vol. 6468, pp. $64680 \mathrm{Q}-64680 \mathrm{Q}-8,2007$.

[56] H. Horie, N. Arai, Y. Yamamoto, and S. Nagao, "Longitudinal-mode characteristics of weakly index-guided buried-stripe type 980-nm laser diodes with and without substrate-mode-induced phenomena," IEEE J. Quantum Electron., vol. 36, no. 12, pp. 1454-1461, 2000.

[57] N. Stelmakh and M. Flowers, "Measurement of spatial modes of broadarea diode lasers with 1-GHz resolution grating spectrometer," IEEE Photon. Technol. Lett., vol. 18, no. 15, pp. 1618-1620, 2006.

[58] P. Crump, S. Böldicke, C. Schultz, H. Ekhteraei, H. Wenzel, and G. Erbert, "Experimental and theoretical analysis of the dominant lateral waveguiding mechanism in $975 \mathrm{~nm}$ high power broad area diode lasers," Semicond. Sci. Technol., vol. 27, no. 4, p. 045001, 2012.

[59] C. Z. Ning, R. A. Indik, and J. V. Moloney, "Effective Bloch equations for semiconductor lasers and amplifiers," IEEE J. Quantum Electron., vol. 33, no. 9, pp. 1543-1550, Sep 1997

[60] M. Lichtner, M. Radziunas, U. Bandelow, M. Spreemann, and H. Wenzel, "Dynamic simulation of high brightness semiconductor lasers," Proc. NUSOD '08, pp. 65-66, 2008.

[61] P. K. Jakobsen, J. V. Moloney, A. C. Newell, and R. Indik, "Space-time dynamics of wide-gain-section lasers," Phys. Rev. A, vol. 45, no. 11, p. 8129,1992

[62] C. Z. Ning, R. A. Indik, J. V. Moloney, W. W. Chow, A. Girndt, S. W. Koch, and R. H. Binder, "Incorporating many-body effects into modeling of semiconductor lasers and amplifiers," Proc. SPIE, vol. 2994, pp. 666-677, 1997.

[63] E. Gehrig and O. Hess, "Spatio-temporal dynamics of light amplification and amplified spontaneous emission in high-power tapered semiconductor laser amplifiers," IEEE J. Quantum Electron., vol. 37 , no. 10 , pp. $1345-1355$, Oct 2001

[64] S. Balsamo, F. Sartori, and I. Montrosset, "Dynamic beam propagation method for flared semiconductor power amplifiers," IEEE J. Quantum Electron., vol. 2, no. 2, pp. 378-384, Jun 1996.

[65] M. Kolesik and J. V. Moloney, "A spatial digital filter method for broadband simulation of semiconductor lasers," IEEE J. Quantum Electron., vol. 37, no. 7, pp. 936-944, Jul 2001

[66] J. Javaloyes and S. Balle, "Quasiequilibrium time-domain susceptibility of semiconductor quantum wells," Phys. Rev. A, vol. 81, no. 6, p. 062505,2010
[67] W. Joyce, "Current-crowded carrier confinement in double-heterostructure lasers," J. Appl. Phys., vol. 51, no. 5, pp. 2394-2401, 1980.

[68] H. Wünsche, U. Bandelow, and H. Wenzel, "Calculation of combined lateral and longitudinal spatial hole burning in $\lambda / 4$ shifted DFB lasers," IEEE J. Quantum Electron., vol. 17, pp. 1751-1760, 1993.

[69] P. Eliseev, A. Glebov, and M. Osinski, "Current self-distribution effect in diode lasers: analytic criterion and numerical study," IEEE J. Sel. Topics Quantum Electron., vol. 3, no. 2, pp. 499-506, 1997.

[70] W. Joyce, "Carrier transport in double-heterostructure active layers," $J$. Appl. Phys., vol. 53, no. 11, pp. 7235-7239, 1982

[71] G. H. B. Thompson, "A theory for filamentation in semiconductor lasers including the dependence of dielectric constant on injected carrier density," Opto-electronics, vol. 4, pp. 257-310, 1972.

[72] D. Mehuys, R. Lang, M. Mittelstein, J. Salzman, and A. Yariv, "Selfstabilized nonlinear lateral modes of broad area lasers," IEEE $J$ Quantum Electron., vol. 23, no. 11, pp. 1909-1920, 1987.

[73] R. Lang, A. Larsson, and J. Cody, "Lateral modes of broad area semiconductor lasers: Theory and experiment," IEEE J. Quantum Electron., vol. 27, no. 3, pp. 312-320, 1991.

[74] S. Blaaberg, P. Petersen, and B. Tromborg, "Structure, stability, and spectra of lateral modes of a broad-area semiconductor laser," IEEE $J$ Quantum Electron., vol. 43, no. 11, pp. 959-973, 2007.

[75] J. V. Moloney, M. Kolesik, J. Hader, and S. W. Koch, "Modeling high-power semiconductor lasers: from microscopic physics to device applications," Proc. SPIE, vol. 3889, pp. 120-127, 2000.

[76] H. Wenzel, P. Crump, H. Ekhteraei, C. Schultz, J. Pomplun, S. Burger, L. Zschiedrich, F. Schmidt, and G. Erbert, "Theoretical and experimental analysis of the lateral modes of high-power broad-area lasers," Proc. NUSOD '11, pp. 143-144, 2011.

[77] M. Spreemann, M. Lichtner, M. Radziunas, U. Bandelow, and H. Wenzel, "Measurement and simulation of distributed-feedback tapered master-oscillator power amplifiers," IEEE J. Quantum Electron., vol. 45, no. 6, pp. 609-616, 2009.

[78] C. Fiebig, V. Tronciu, M. Lichtner, K. Paschke, and H. Wenzel, "Experimental and numerical study of distributed-Bragg-reflector tapered lasers," Appl. Phys. B, vol. 99, no. 1, pp. 209-214, 2010.

[79] C. M. Schultz, P. Crump, H. Wenzel, O. Brox, A. Maaßdorf, G. Erbert, and G. Tränkle, " $11 \mathrm{~W}$ broad area $976 \mathrm{~nm}$ DFB lasers with $58 \%$ power conversion efficiency," Electron. Lett., vol. 46, no. 8, pp. 580-581, 2010 .

[80] H. Wenzel, G. Erbert, and P. Enders, "Improved theory of the refractiveindex change in quantum-well lasers," IEEE J. Sel. Topics Quantum Electron., vol. 5, no. 3, pp. 637-642, 1999.

[81] J. Pomplun, H. Wenzel, S. Burger, L. Zschiedrich, M. Rozova, F. Schmidt, P. Crump, H. Ekhteraei, C. Schultz, and G. Erbert, "Thermo-optical simulation of high-power diode lasers," Proc. SPIE, vol. 8255, p. 24, 2012.

[82] J. Moloney, "Semiconductor laser device modeling," in Fundamental Issues of Nonlinear Laser Dynamics, B. Krauskopf and D. Lenstra, Eds. American Institut of Physics, 2000, pp. 149-172.

[83] K. Böhringer, "Microscopic spatio-temporal dynamics of semiconductor quantum well lasers and amplifiers," Ph.D. dissertation, Institut für Technische Physik, Deutsches Zentrum für Luft- und Raumfahrt, 2007.

[84] I. Fischer, O. Hess, W. Elsäßer, and E. Göbel, "Complex spatiotemporal dynamics in the near-field of a broad-area semiconductor laser," Europhys. Lett., vol. 35, no. 8, pp. 579-584, 1996.

[85] G. Wachutka, "Rigorous thermodynamic treatment of heat generation and conduction in semiconductor device modeling," IEEE Trans. Comput.-Aided Design Integr. Circuits Syst., vol. 9, no. 11, pp. 11411149, 1990.

[86] Z. Li, K. Dzurko, A. Delage, and S. McAlister, "A self-consistent twodimensional model of quantum-well semiconductor lasers: optimization of a GRIN-SCH SQW laser structure," IEEE J. Quantum Electron., vol. 28, no. 4, pp. 792-803, 1992.

[87] B. Witzigmann, A. Witzig, and W. Fichtner, "A multidimensional laser simulator for edge-emitters including quantum carrier capture," IEEE Trans. Electron Devices, vol. 47, no. 10, pp. 1926-1934, 2000.

[88] U. Bandelow, H. Gajewski, and R. Hünlich, "Fabry-Perot lasers: Thermodynamics based modeling," in Optoelectronic Devices - Advanced Simulation and Analysis, J. Piprek, Ed. New York: Springer, 2005, pp. 63-85.

[89] H. Wenzel and G. Erbert, "Simulation of single-mode high-power semiconductor lasers," Proc. SPIE, vol. 2693, pp. 418-429, 1996.

[90] [Online]. Available: http://www.wias-berlin.de/software/tesca/

[91] H. Wenzel, P. Crump, A. Pietrzak, C. Roder, X. Wang, and G. Erbert, "The analysis of factors limiting the maximum output power of broad- 
area laser diodes," Opt. Quantum Electron., vol. 41, no. 9, pp. 645-652, 2009.

[92] H. Wenzel, P. Crump, A. Pietrzak, X. Wang, G. Erbert, and G. Tränkle, "Theoretical and experimental investigations of the limits to the maximum output power of laser diodes," New J. Phys., vol. 12, no. 8, p. 085007, 2010.

[93] B. S. Ryvkin and E. A. Avrutin, "Asymmetric, nonbroadened large optical cavity waveguide structures for high-power long-wavelength semiconductor lasers," J. Appl. Phys., vol. 97, no. 12, pp. $123103-$ $123103,2005$.

[94] _ "Effect of carrier loss through waveguide layer recombination on the internal quantum efficiency in large-optical-cavity laser diodes," J. Appl. Phys., vol. 97, no. 11, pp. 113 106-113 106, 2005.

[95] — " "Spatial hole burning in high-power edge-emitting lasers: A simple analytical model and the effect on laser performance," J. Appl. Phys., vol. 109, no. 4, pp. 043 101-1-043 101-5, 2011.

[96] G. Albinus, H. Gajewski, and R. Hünlich, "Thermodynamic design of energy models of semiconductor devices," Nonlinearity, vol. 15, no. 2, p. 367, 2002.

[97] Z. Li, S. McAlister, and C. Hurd, "Use of Fermi statistics in twodimensional numerical simulation of heterojunction devices," Semiconductor science and technology, vol. 5, no. 5, pp. 408-413, 1990.

[98] M. Grupen, K. Hess, and G. Song, "Simulation of transport over heterojunctions," in Proc. 4th Int. Conf. Simul. Semicon. Dev. Process, vol. 4, 1991, pp. 303-311.

[99] S. Steiger, R. Veprek, and B. Witzigmann, "Unified simulation of transport and luminescence in optoelectronic nanostructures," J. Comp. Electron., vol. 7, no. 4, pp. 509-520, 2008.

[100] M. Grupen and K. Hess, "Simulation of carrier transport and nonlinearities in quantum-well laser diodes," IEEE J. Quantum Electron., vol. 34, no. 1, pp. 120-140, 1998.

[101] L. Borruel, J. Arias, B. Romero, and I. Esquivias, "Incorporation of carrier capture and escape processes into a self-consistent cw model for quantum well lasers," Microelectron. J., vol. 34, no. 5, pp. 675-77, 2003.

[102] Y. Liu, W. Ng, K. Choquette, and K. Hess, "Numerical investigation of self-heating effects of oxide-confined vertical-cavity surface-emitting lasers," IEEE J. Quantum Electron., vol. 41, no. 1, pp. 15-25, 2005.

[103] N. Tansu and L. J. Mawst, "Current injection efficiency of InGaAsN quantum-well lasers," J. Appl. Phys., vol. 97, no. 5, pp. $054502-$ $054502,2005$.

Hans Wenzel received the Diploma and Doctoral degrees in physics from Humboldt-University Berlin, Germany, in 1986 and 1991, respectively. His thesis dealt with the electro-optical modeling of semiconductor lasers. From 1991 to 1994, he was involved in a research project on the simulation of distributed feedback lasers. In 1994, he joined the Ferdinand-BraunInstitut, Leibniz-Institut für Höchstfrequenztechnik, where he is engaged in the development of high-brightness semiconductor lasers. He authored or coauthored more than 250 journal papers and conference contributions. His main research interests include the analysis, modeling, and simulation of optoelectronic devices. 